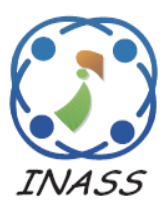

\title{
Reliability Evaluation of Electric Distribution Network with Distributed Generation Integrated
}

\author{
Ali Mohammed Jaleel ${ }^{1}$ \\ Mohammed Kdair Abd ${ }^{1 *}$ \\ ${ }^{I}$ Department of Electrical Engineering, University of Technology - Iraq, Baghdad, Iraq \\ * Corresponding author's Email: $30098 @$ uotechnology.edu.iq
}

\begin{abstract}
The electrical power system, especially that of the Electric Distribution Network (EDN) is more complex for the rapid deployment and penetration of Distributed Generation (DG). The DGs in the EDN are vulnerable to faults, and the reliability index considered is a critical factor in the work continuation of the EDN. The Particle Swarm Optimization (PSO) is modified to restrict the particle velocities when it runs to obtain the optimum solution for DG placement and capacity in the distribution network. This modification prevents the velocities from reach an acceptable level within a few iterations. This paper presents a new approach and good analysis to the evaluation of reliability and estimates the optimal location and capacity of the DGs units with multi-objective functions for power loss reduction and improves voltage profile. The optimization approach is based on the new Modified Particle Swarm Optimization (MPSO) for decision-making on strategic distribution system points for location and capacity of DGs using Matlab software.In this study, reliability is evaluated using the "Electrical Transient Analyzer Program, ETAP" and applied on an IEEE 33-bus test system. The obtained results of the proposed approach show superior on the other methods a reduction in real power losses by $(60.13 \%)$ and an improvement in voltage profile by $(88.34 \%)$.
\end{abstract}

Keywords: Reliability index, Distributed generator, PSO, Electrical transient analyzer, Power losses.

\section{Introduction}

Power losses, energy efficiency issues, low voltage profiles [1], and consumer service issues are just a few of the challenges that EDN face. These problems can be solved by adding DGs optimally to the EDN [2], system reconfigurations [3], and finding solutions to reduce reliance on fossil fuels by utilizing renewable energy sources [4]. In power transmission and distribution systems, electrical energy management and pollution reduction are critical issues, and Economic Dispatch (ED) is being used to address these issues [5]. There is no definitive definition that encompasses all DG, so DGs are small-scale decentralized generators directly connected to a power grid at the distribution voltage level and its value usually depends on the network capacity. This often discusses network issues such as service availability, losses, and voltage profile. DGs, also known as embedded distributed generation or decentralized generation, are sources of electricity directly connected to the EDN or even on the consumer's side of the meter. Inappropriate placement and size of DGs in the EDN may have the following negative consequences: Risings in the short circuit current levels, the voltage becomes set out of reasonable limits, lines may have overloading, and power loss increase. DGs have several benefits that have been noted, including certain drawbacks.

Many of these benefits include Peak load saving, reliability and voltage stability improvement, decreasing power losses, power quality enhancement and less polluting emissions, improved security, grid strengthening, and reduced on-peak operating cost [6]. The demand for electrical service reliability is growing in modern society. Therefore, the distribution system's reliability has gained more attention as an important concern that needs to address to provide customers with a higher level of trust. In EDN, reliability evaluation means determining the system's ability to meet the load's demands. The reliability of each variable used in the 
system determines the overall system's reliability. Each element has two states; on and off. By deciding whether a component is operating or not.

The configuration and operating characteristics of the entire power system are reflected in distribution system reliability. According to reports, distribution system failure is responsible for more than $80 \%$ of consumer power outages [7]. Since it is the weakest connection between the source of supply and the consumer load points, the distribution system has the most significant impact on supply reliability. Among the many research and papers published in this area; the optimal placement and capacity of distributed energy storage systems are determined using a mixed-integer second-order cone programming model. It is demonstrated on an IEEE 33-bus distribution network. One potential drawback of the proposed methodology is that the computational cost of solving the optimization problem could increase as the number of design variables rises, making largescale challenges impossible to address [8].

In order to minimize the power loss and voltage profile improvement using a loss reduction sensitivity factor (LRSF) to find the optimum DG placement while the size of the DG is calculated using the New Enhanced Symbiotic Organisms Search (NeSOS) method. The traditional SOS algorithm problems: lack of organism variability, inefficient computational time, and an imbalance of exploration and exploitation [9]. In reference [10], models for utility-based DG penetration in radial distribution systems are both optimal and maximum. Therefore, several problems with different probabilistic indices as objective functions constrained by power flow equations, DGs penetration, voltage, and thermal limits are proposed. The optimum DG placement and size were determined using a novel approach. The DGs are installed in the primary EDN to reduce power losses and improve voltage stability using the PSAT toolbox. The PSAT toolbox has the disadvantage of requiring a pricey license, and making changes to the models and algorithms provided with the program is often difficult [11].

In [12], an offline-online approach for DG placement and sizing in the EDN divides the solution of the related parametric power flow problem and optimization into separate stages. The proposed method drawback, offline and online phases separately treated, offline decisions are made without regard for the downstream online solver's abilities, while the applicability of the best methods for online decisions is restricted by the need for high responsiveness. DGs placement in the EDN improves bus voltage profile and minimizes power loss, applied in [13] based on an integrated voltage stability index and (Dragonfly) algorithm. Different case studies are simulated using the proposed approach on the standard IEEE 83-bus test system. One of the Dragonfly algorithm drawbacks; lack of internal memory, which causes its premature convergence to the local optimal. In reference [14] Employing Biogeography-based optimization (BBO) to determine the best position and sizing for DG units to minimize power losses and controlling voltage (profile and harmonic distortion) within acceptable limits. In [15] analyzes the options for improving the reliability of microgrids with a high presence of renewable generation by addressing variable renewable generation constraints. Load shedding, energy storage, DGs, and the creation of smaller microgrids within a microgrid are all investigated. Show the impact of DG placement in distribution system networks on reliability worth [16].

Different reliability indices, such as SAIDI, CAIDI, EENS, and ASAI, are used to measure the improvement in reliability. The reliability of EDN with DG sources has been assessed using the PSO approach is used to deal with complex formulations [17]. The focusing on system uncertainties and the appropriate restoration strategies. The restoration optimization formulation for reliability improvement has taken into account the uncertainties associated with; (i) Renewable energy power output, (ii)Timevarying load demand, and (iii) Stochastic prediction errors and random fault events. In [18] employed PSO approach in the EDN to find the best placement and capacity of DG for increase reliability indices, reduce real power loss, and improve the voltage profile. Furthermore, the encoded Markov cut set algorithm is used to test reliability indices.

Reference [19] presents an integrated approach for evaluating the effect that Distributed Energy Resources (DERs) can have on power network reliability. The traditional Monte Carlo approach is updated to include time-varying electricity demand profiles and network component failure rates. For reliability evaluation, reference [20] proposes a combination of Monte Carlo simulation and the $2 \mathrm{~m}$ point estimate process, and the Genetic Algorithm (GA) is used to reduce the penalty-taking cognizance of investment cost by using the reliability tool to find optimal location and size of DG. The GA algorithm drawback is that the encoding and decoding procedures can take a long time to compute. As well, the Modified Gbest-guided artificial bee colony meta-heuristic proposed method was updated and applied to solve the problem of DG placement to improve reliability, index of voltage stability, and 
lower operating costs, voltage variance, and power losses [21].

The Teaching and Learning Based Optimization (TLBO) [22] and symbiotic organisms search (SOS) [23] calculate the best placement for multiple DG in the EDN to minimize the power loss, increase reliability, and improve voltage profile. Improved PSO (IPSO) and Bat Inspired Algorithms have been used to determine the best size and position for DG units in EDN [24]. For IEEE 33-bus and IEEE 69-bus test systems, distributed load flow is used to calculate power system losses and voltage at each bus. The Ant lion optimizer is used in [25] to decide the optimum DG placement and size for improving voltage profiles in the radial EDN. For power losses and bus voltage profile enhancement, in [26] proposed a new master-slave hybrid technique based on both the parallel PBIL (PPBIL) algorithm and the PSO. The parallel implementation of the Population-Based Incremental Learning (PBIL) method was used for optimal DG placement, and Particle Swarm Optimization has been used for optimal capacity. The Loss Sensitivity Factor (LSF), a Genetic Technique (GA), and a Parallel Monte-Carlo algorithm (PMC) are all compared to the proposed technique.

A particle swarm is an algorithm that uses the interaction of individuals in a group of particles to find optimal areas for complex search spaces. Therefore, the researchers were not able to elucidate it well. Traditional versions of the algorithm have undesirable dynamic properties, particularly particle velocities, which must be restricted to regulate their paths. The particle's path is analyzed in separate time "Algebraic display" before progressing to its width in continuous time "Analytical view". These analyzes result in a generalized algorithm model with a set of parameters for controlling system convergence trends. Some of the particle swarm optimizer results and implementation modifications derived from the analysis suggest ways to change the original algorithm to eliminate problems and improve the particle swarm's ability to optimize some wellstudied test functions. These modifications in this work yielded promising results in determining the location and size of the generators, thereby improving the reliability.

This paper employed the new Modified Particle Swarm Optimization (MPSO) depending on the modification of particle velocity to find the optimal placement and size of the DGs in the EDN. In this study, Three criteria are used to assess the effectiveness of the suggested approach: active power loss reduction, voltage profile improvement, and reliability enhancement. In contrast to the PPBIL, PMC, GA, and LSF methods [26], the results show that the new MPSO provides the optimum balance between voltage profiles, power loss reduction, and reliability enhancement. In addition, different scenarios by fixing DGs in suitable locations and generating power based on the load conditions are presented to verify and performance of the proposed method compared with other methods. An IEEE 33bus test system was selected to carry out the different scenarios.

The rest of the paper is organized as follows. Section 2 presents the problem formulation and the objective function. Then, section 3 describes the PSO, and section 4 introduces the reliability of the power system. Section 5 includes results and discussion. Finally, the conclusions in section 6 .

\section{Problem formulation}

Distribution systems suffer from high power losses, low voltage levels, high current, and low reliability due to increase load demand. Therefore to improve these problems by incorporating DGs into the delivery system. One of the most common techniques for reliability enhancement, power loss reduction, and voltage profile improvement is optimal DG placement in the distribution system. Therefore, selecting the optimal placement and size of the DGs is needed to function appropriately in the EDN.

\subsection{Load flow}

The analysis of load flow is an important aspect of power system studies because of their radial topology and high $(\mathrm{R} / \mathrm{X})$ ratio and the NewtonRaphson and Gauss-Seidel are failed in radial distribution networks. This study is dependent on backward/forward sweep processes using Kirchhoff's rules [27].

\subsection{Objective function (K)}

By incorporating DGs for a multi-objective on the distribution grid, the goal is to minimize actual power losses and voltage profile improvement. After that, the reliability indexes are assessed by determining the optimal DG placement and size. The objective functions $(K)$ can be expressed in the following equations:

$$
\begin{gathered}
K=C S_{1}+S_{2}(1-C) \\
S_{1}=\frac{P_{T L, w i t h ~ D G}}{P_{T L, w i t h o u t ~ D G}}
\end{gathered}
$$




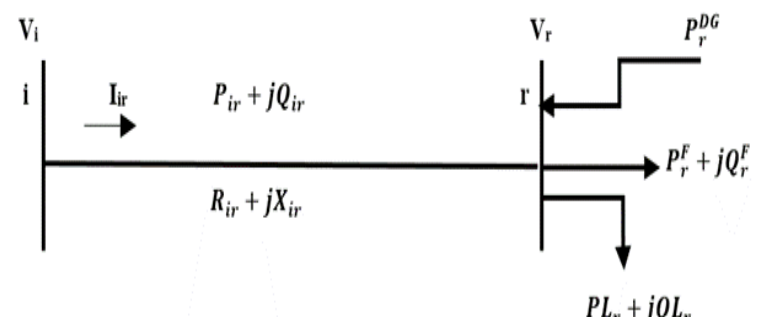

Figure. 1 Equivalent branch of an electrical network

$$
S_{2}=\frac{\operatorname{Ver}_{\text {with } D G}}{\left(\text { Ver }_{\text {without } D G}\right)(n)}
$$

Where: $\mathrm{S} 1$ is the percentage of total active loss with DGs compared to what was previously, $\mathrm{S} 2$ is the average ratio of (Ver) at each bus with DGs to total (Ver) before adding DGs, PTL,withDG is real power losses after adding DG, PTL, withoutDG is real power losses before adding DGs, VerwithDG is voltage profiles square error after adding DGs, Verwithout DG is voltage profiles square error before adding DGs, $\mathrm{n}$ is the number of buses and $\mathrm{C}$ the weight factor $(1 \geq \mathrm{C} \geq 0)$.

\subsection{Voltage profiles square error (Ver)}

When the square error in the voltage profiles is being reduced, then the voltage profile of the system is improved. Square error in the voltage profiles can be calculated as shown in Eq. (4)

$$
\operatorname{Ver}=\sum_{j=1}^{n}\left(V_{i}-V_{r e f}\right)^{2}
$$

Where: Vi is bus voltages at nodes i, Vref is the reference voltage and Equals to 1 p.u.

\subsection{Reliability indices}

The indices are evaluated for divergent DGs reliability by finding the optimal size and location of the DGs. Besides that, the distribution network's reliability has been improved by combining one DG with multiple DG in the distribution system. Some of the reliability indices used to assess a system's reliability are EENS, AENS, SAIDI, SAIFI, and ASAI. They were also used in this study to assess the improvement in reliability.

\subsection{Constraints}

\subsubsection{Power flow calculation}

The line segment is known to have series impedance $\left[R_{i r}+j X_{i r}\right]$. The active and reactive $\left[P_{L r}+j Q_{L r}\right]$ load power, as shown in Fig. 1. The flow of active and reactive power between buses is calculated using Eqs. (5) and (6). The Vr end bus voltage reception is determined using Eq. (7) [21]. With the help of Kirchoff's law, the set of Eqs. (5) to (10) was derived.

$$
\begin{gathered}
P_{i r}=P_{r}^{F}+P L_{r}-P_{r}^{D G}+\frac{R_{i r}}{V_{i}^{2}}\left(P_{i r}^{2}+Q_{i r}^{2}\right) \\
Q_{i r}=Q_{r}^{F}+Q L_{r}+\frac{X_{i r}}{V_{i}^{2}}\left(P_{i r}^{2}+Q_{i r}^{2}\right) \\
V_{r}^{2}=V_{i}^{2}-2\left(P_{i r} R_{i r}+Q_{i r} X_{i r}\right)+ \\
\frac{R_{i r}^{2}+X_{i r}^{2}}{V_{i}^{2}}\left(P_{i r}^{2}+Q_{i r}^{2}\right)
\end{gathered}
$$

Moreover, the current flowing through an Iir branch is measured using Eq. (8):

$$
I_{i r}^{2}=\frac{P_{i r}^{2}+Q_{i r}^{2}}{V_{i r}^{2}}
$$

The power loss of any bus connecting portion of the line $(i)$ and $(r)$ as show in Eq. (9):

$$
P_{L O S S}(i, r)=I_{i r}^{2} R_{i r}
$$

The description of these power losses in each branch illustrates the network's total power loss, and it can be described using Eq. (10);

$$
P_{T \text { LOSS }}=\sum_{\substack{i=0 \\ i \neq r}}^{n-1} P_{\text {LOSS }}(i, r)
$$

\subsubsection{Power balance}

The active and reactive power balance expressions are shown in Eqs. (11) and (12).

$$
\begin{array}{r}
P_{n e t_{i}}=P_{D G_{i}}-P_{d m_{i}}-v_{i} \sum_{r=1}^{n} v_{r} y_{i, r} \cos \left(\delta_{i}-\delta_{r}\right. \\
\left.-\theta_{i}+\theta_{r}\right) \\
Q_{n e t_{i}}=Q_{D G_{i}}-Q_{d m_{i}}-v_{i} \sum_{r=1}^{n} \begin{array}{r}
v_{r} y_{i, r} \sin \left(\delta_{i}-\delta_{r}\right. \\
\left.-\theta_{i}+\theta_{r}\right)
\end{array}
\end{array}
$$

Where: $P_{\text {neti }}$ and $Q_{\text {neti }}$ are the net active and reactive power at $i$-bus, and equal to zero, $P_{D G i}$ and $Q_{D G i}$ are the active and reactive power at the $i$-bus for DGs, $P_{d m i}$ and $Q_{d m i}$ are the active and reactive load demands, $V_{r}$ is the bus voltage at the $r$-bus, $Y_{i, r}$ is the branch admittance between the $i$ and $r$-buses, $\delta \mathrm{i}$ and $\delta \mathrm{r}$ are the phase angles of the $i$-bus and $r$-bus voltages, $(\theta \mathrm{i}$ $\theta \mathrm{r})$ are the impedance angle of the branch connecting $i$ and $r$-buses. 


\subsubsection{DGs capacity}

These constraints ensure the non-reversal of power flow. The power supplied by the substation to the distribution system should be higher than the DGs power. The DGs also have the power generation min and max limits [28].

$$
P^{D G} \min \leq P^{D G} \leq P^{D G} \max
$$

Where: $P^{D G}$ is the DGs active power, $P^{D G} \mathrm{~min}$ : Zero, $P^{D G} \max$ is the max DGs power.

\subsubsection{Voltage limitation}

Each bus voltage should be between the min and max ranges.

$$
0.95 \text { p.u. } \leq V m \leq 1.05 \text { p.u. }
$$

\subsubsection{Branch current}

The current in the distribution system lines should be regulated and must not surpass the maximum current.

$$
I i \leq I_{\max , I}
$$

\section{Particle swarm optimization (PSO)}

\subsection{Standard PSO}

PSO Technique will carry out DGs optimum number and location in this case study. In 1995, Eberhart and Kennedy [29, 30] developed this method. The PSO algorithm was based on the social behaviour of organisms like fish schooling and birds flocking. PSO provides a population-based method of searching people called particles for a period to adjust their location. Speed and location for each particle adjusted by following Eqs. (16) and (17) during each iteration of the algorithm until the stop criteria have been met.

$$
\begin{array}{r}
V_{i}^{k+1}=W V_{i}^{k}+c_{1} r_{1}\left(X_{\text {pbest }_{i}^{k}}-X_{i}^{k}\right)+ \\
+c_{2} r_{2}\left(X_{\text {Gbest }^{k}}-X_{i}^{k}\right) \\
X_{i}^{k+1}=X_{i}^{k}+V_{i}^{k+1}
\end{array}
$$

Where: $V_{i}^{k}$ is the particle $i$ velocity at iteration $k$, $X_{i}^{k}$ is the current particle $i$ at iteration $k, X_{\text {pbest }_{i}^{k}}$ and $X_{\text {Gbest }^{k}}$ these are respectively the best fitness values and best values for any particle in the population, $r_{l}$ and $r_{2}$ is the random number between $(0,1)$ and $c_{1}, c_{2}$ acceleration constants.

\subsection{New modified particle swarm optimization (MPSO)}

If particulate speeds are not restricted when PSO runs, the speeds can increase in a few iterations to unacceptable levels. The introduction of constraint coefficients for the regulation of particulate speeds therefore modified this approach. The coefficient controls and directs the particle movements to convergence. Modified particles velocity can be shown as follows:

$$
\begin{gathered}
V_{i}^{k+1}=W_{\text {new }} V_{i}^{k}+c_{1 \text { new }} r_{1}\left(X_{\text {pbest }}^{k}-X_{i}^{k}\right) \\
+c_{2 \text { new }} r_{2}\left(X_{\text {Gbest }}{ }^{k}-X_{i}^{k}\right) \\
X_{i}^{k+1}=X_{i}^{k}+V_{i}^{k+1}
\end{gathered}
$$

Where: $c_{\text {lnew }}, c_{2 \text { new }}$ are cognitive and social elements that influence convergence speed and search space for optimal point. The $c_{1}$ and $c_{2}$ can be written as follow:

$$
\begin{gathered}
c_{1 \text { new }}=(D F) \Delta 1, c_{2 \text { new }}=(D F) \Delta 2 \\
W_{\text {new }}=W(D F)
\end{gathered}
$$

Modify Eq. (18) to improve PSO performance, as particle velocities and spatial coordinates careen towards infinity, the PSO random weighting of control parameters generates an explosion. These constriction coefficients can avoid explosions and can also cause particles to converge on local optimums. The constriction factor (DF) can be written as follow:

$$
D F=\frac{2}{\left|\Delta-2+\left(\sqrt{\left|\Delta^{2}-4 \Delta\right|}\right)\right|}\left(1-\left(\frac{h}{H}\right)^{2}\right)^{2}
$$

Where: $W$ is inertia weight, $\Delta=\Delta 1+\Delta 2, \Delta 1+$ $\Delta 2 \geq 4, \Delta$ is the co-efficient and equal to $4.1, \Delta 1$ equal to $\Delta 2, H$ is the number of iterations, and $h=1$, $2,3, \ldots, H$. Without using velocity boundaries, the constriction particles can bring the process closer to the optimum solution.

\subsection{The MPSO model}

In the MPSO model, the particulate speeds are restricted by the introduction of constraint coefficients. The coefficient controls and directs the particle movements to convergence. The steps of the proposed MPSO model can be described as follows: Step1: Input system data (line and bus data). 
Step2: Create an initial population of particles with random positions and velocities on dimensions in the solution space (random number and position of DGs). Set the iteration counter to 0 .

Step3: Calculate the power loss for the base case by using load flow.

Step4: For each particle, compare the objective value to the best individual. If the objective value is less than XPbest, set this value to the current XPbest and save the particle position.

Step5: Set the value of Xgbest to the particle that is associated with the lowest Xpbest.

Step6: Update the particle velocity and position using Eqs. (18) and (19).

Step7: If the iteration number reaches the total limit, proceed to step 9. If not, return to Step 4 and set the iteration index to $h=h+1$

Step8: Create a printout of the best solution, and this is the perfect solution for the delivery systems optimum DGs positioning and sizing. Fig. 2 shown the flow chart of MPSO model.

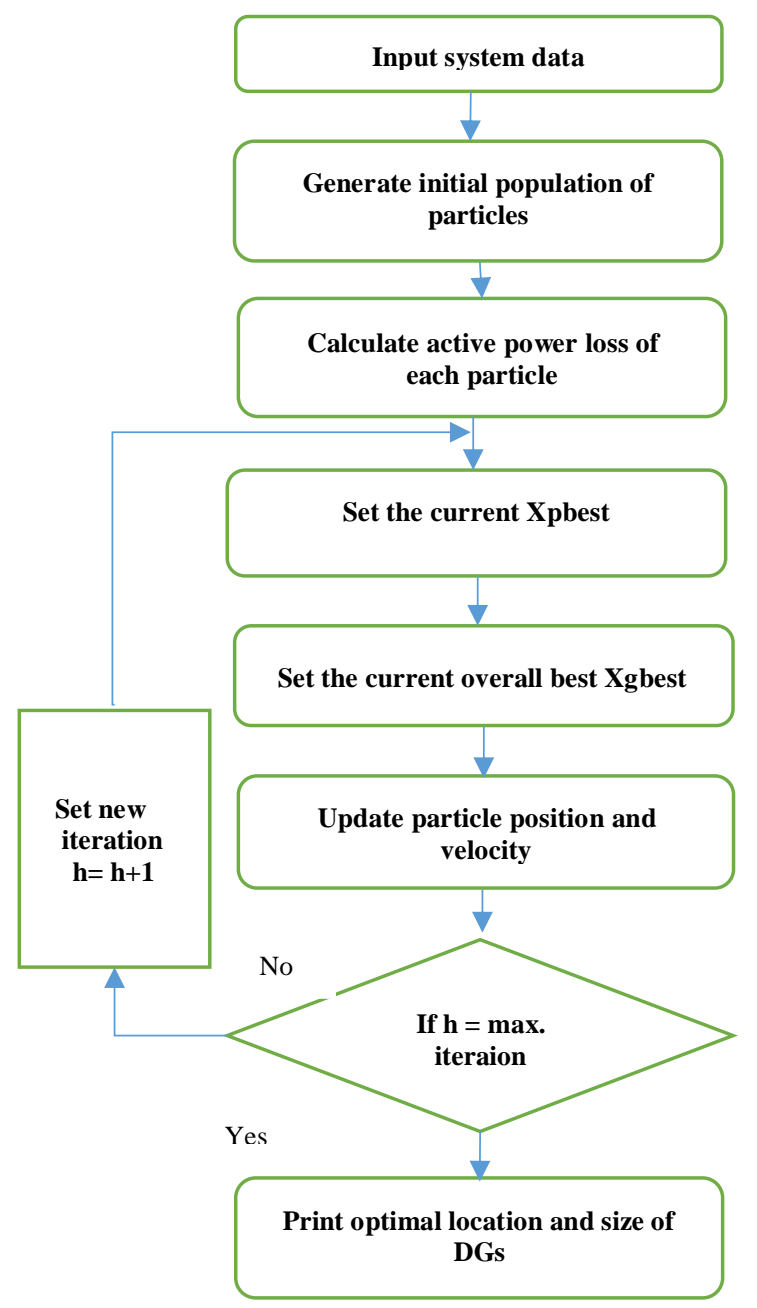

Figure. 2 Flow chart of MPSO model

\section{Reliability of power system}

For its customers, reliability is essential. Any state of supplied electricity that causes a fault in equipment or a system is described as a power quality problem from the consumer's viewpoint. The utility would be able to do its job to the best of its ability to provide adequate voltage to its customers. Utilities are usually unconcerned about the current received by end-users. An ideal sinusoid with unvarying frequency and amplitude is needed for perfect power quality. The capacity of electricity generating stations to provide consumers with continuous electricity is referred to as system adequacy. The following basic requirements for system adequacy must be met to ensure system adequacy: a- In addition to the load demand, a plant generates capacity that should always be higher than total system power loss, b- The system must be capable of transporting load demand to the consumer end without disrupting equipment, cTo provide service to the customer within a specific voltage range. An acceptable voltage range based on the number of customers must be served.

\subsection{Components modeling}

The reliability indices are commonly used to assess EDN reliability [31], as shown as follow:

1. Average Failure Rate $(\lambda)$ : the load interruption frequency. The sum of active $(\lambda a)$ and passive $(\lambda b)$ failure rates is the average failure rate.

2. Mean Time to Repair (MTTR): the time (in hours) required to restore an element outage to its normal operation. It is equal to $r$.

$$
M T T R=r
$$

3. Average Repair Rate $(\mu)$ : Frequency of repair and occurrence per year.

$$
\mu=\frac{8760}{M T T R}
$$

4. Mean Time to Failure (MTTF): The estimated amount or (proper) of time (in years) that the component will remain in a failed state.

$$
M T T F=\frac{1}{\lambda}
$$

5. Mean Time Between Failures $(M T B F)$ : Expected time in years a component fails. The differences between MTTR, MTTF, and MTBF are shown in Fig. 3.

$$
M T B F=M T T F+M T T R / 8760
$$




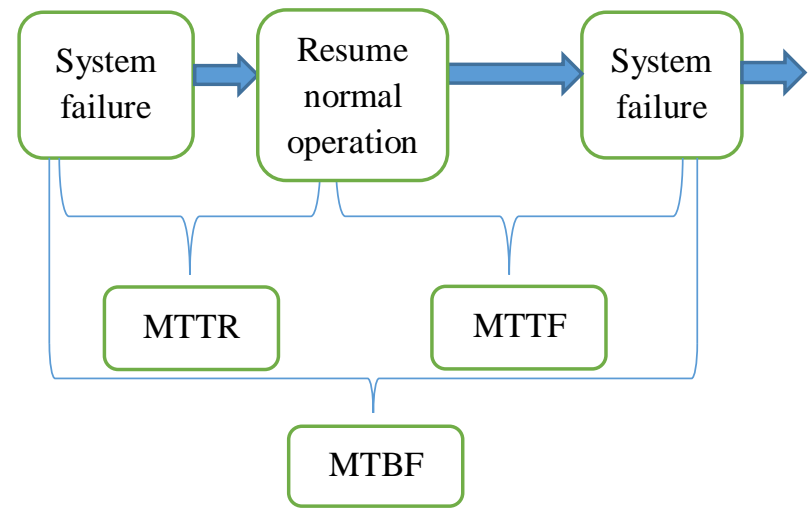

Figure. 3 Differences between MTTR, MTTF and MTBF

\subsection{Solar photovoltaic (SPV)}

A solar photovoltaic model was used in the reliability assessment of the EDN, which is modelled by the ETAP program. This work employed the Sunniva ART245-60-3-1 module of 240WP. The characteristics of the ART245-60-3-1 are taken under STC (Standard Test Conditions) in the laboratory environment. $1000 \mathrm{~W} / \mathrm{m} 2$ irradiation, $25^{\circ} \mathrm{C}$, and 1.17 solar spectrum air mass are the default conditions. Table 1 shows the details of the SPV module that was used in this analysis.

$$
\begin{gathered}
\text { Eff }=\text { Power } /(A \times \text { Irradiance }) \\
F F=\frac{\text { Power }}{V_{o c} \times I_{s c}} \%
\end{gathered}
$$

Where: $A$ is the area of PV array, (Eff) panel efficiency in $\%,(F F)$ panel fill factor in per cent, $V_{o c}$ and $I_{s c}$ are the open-circuit voltage and short-circuit current, respectively.

Table 1. PV (Sunniva ART245-60-3-1) specifications

\begin{tabular}{|l|c|}
\hline Parameter & Rating (Unit) \\
\hline Max power & $240 \mathrm{~W}$ \\
\hline Vmp (voltage at max power) & $30.65 \mathrm{~V}$ \\
\hline Imp (current at max power) & $7.82 \mathrm{~A}$ \\
\hline Voc (open circuit voltage) & $37.08 \mathrm{~V}$ \\
\hline Isc (short circuit current) & $8.33 \mathrm{~A}$ \\
\hline Eff (panel efficiency) & $14.9 \%$ \\
\hline Tol. P (Tolerance of Power) & $\pm 4.8 / 0 \%$ \\
\hline Fill Factor & $77.6 \%$ \\
\hline Temperature & $25 \mathrm{C}^{\circ}$ \\
\hline $\begin{array}{l}\alpha \text { (adjustment coefficient temperat- } \\
\text { ure for short circuit current) }\end{array}$ & $0.036 \% / \mathrm{C}^{\circ}$ \\
\hline $\begin{array}{l}\beta \text { (adjustment coefficient temperat- } \\
\text { ure for open-circuit voltage) }\end{array}$ & $-0.332 \% / \mathrm{C}^{\circ}$ \\
\hline $\begin{array}{l}\gamma \text { (adjustment coefficient temperat- } \\
\text { ure for power) }\end{array}$ & $-0.465 \% / \mathrm{C}^{\circ}$ \\
\hline
\end{tabular}

\subsection{Modeling of system reliability}

Compared to other components and parts of the distribution system, the reliability assessment is just as important. The IEEE standard number 1366 [32] provides a guide for distribution system reliability. According to a given standard, the reliability of a distribution system can be assessed using some reliability indices. These indices are primarily divided into two groups:

\subsubsection{Load point reliability indices}

1. Average Failure Rate at Load Point (i), $\lambda i$ (failure per year):

$$
\lambda i=\sum_{j \in \mathrm{Ne}} \lambda_{e, j}
$$

Where: $\lambda i$ is average failure rate at load point $(i), N e$ is total number of elements whose fault will interrupt load point $i, \lambda_{e, j}$ is average failure rate.

2. Annual Outage Duration at Load Point (i), Ui (an hour per year):

$$
\mathrm{Ui}=\sum_{\mathrm{j} \in \mathrm{Ne}} \lambda \mathrm{e}, \mathrm{j} \cdot r_{\mathrm{i}, \mathrm{j}}
$$

Where: Ui is annual outage duration at load point $(i)$, $\lambda e, j$ : Average failure rate, $\mathrm{Ne}$ is total number of elements whose fault will interrupt load point $I, r_{i, j}$ is failure duration at load point $(i)$ due to a failed element $j$.

3. Average Outage Duration at Load Point (i), ri (hours):

$$
r i=\frac{\text { Annual outage duration at load point } i, U i}{\text { Average failure rate at load point } i, \lambda i}
$$

\subsubsection{System based indices}

1. System Average Interruption Frequency Index (SAIFI)

$$
S A I F I=\frac{\sum N i}{N T}
$$

Where $N i$ : Total number of interrupted customers, $N T$ : Total number of customers served.

2. System Average Interruption Duration Index (SAIDI).

$$
S A I D I=\frac{\sum r_{i} * N i}{N T}
$$

Where: $r i$ is restoration time in minutes.

3. Customer Average Interruption Duration Index (CAIDI). 


$$
C A I D I=\frac{\sum r_{i} * N i}{N i}
$$

4. Average Service Availability Index (ASAI).

$$
A S A I=\frac{\text { Customer Hours Service Availability }}{\text { Customer Hours Service Demands }}
$$

5. Energy Not Supplied (ENS).

$$
E N S=\sum L_{i} U_{i}
$$

6. Average Energy Not Supplied (AENS).

$$
\text { AENS }=\frac{\text { Total enerage not supplied }}{\text { total No.of customer surved }}
$$

7. Average Service Unavailability Index (ASUI).

$$
A S U I=1-A S A I \text { (p.u) }
$$

\section{Results and discussion}

The optimum DGs placement and size are obtained and analyzed by employed the proposed approach, including distribution system reliability. This analysis considers the 33 bus distribution system as shown in Fig. 4. The branch and load data are taken from [26] for this method. It consists of 33 buses and 32 branches with active power and reactive power loads of a total of (3.715MW) and (2.3MVAr). The distribution system operates at $12.66 \mathrm{kV}$. For the base case, the active power losses are $(0.211 \mathrm{MW})$, and the Ver was $(0.1338$ p.u). The proposed design was implemented using Matlab R2015a programs and executed on a computer with an Intel(R) Core(TM)i7 processor running at $2.5 \mathrm{GHz}$ and $8 \mathrm{~GB}$ of RAM. With three case studies, MPSO is used to determine the best location and size of DGs with unity power factors:

1. Case \#1: allocation and size of single DGs.

2. Case \#2: allocation and size of two DGs.

3. Case \#3: allocation and size of three DGs.

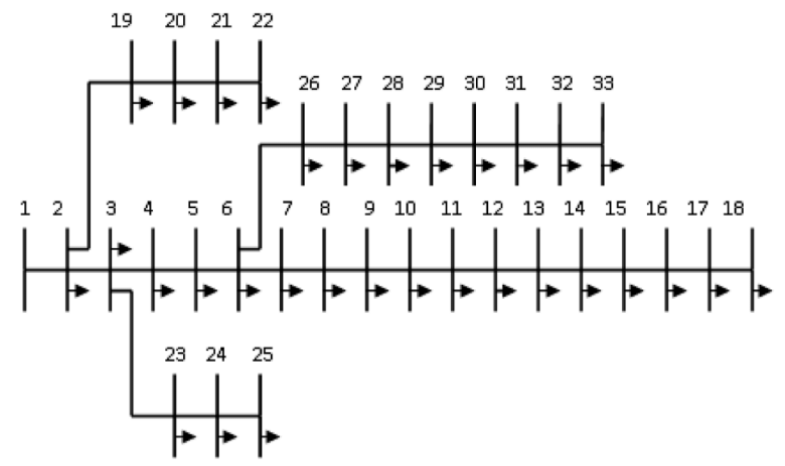

Figure. 4 One-line diagram of IEEE 33-bus test system
The results are obtained by following the instructions below.

a) Power loss reduction and voltage square error are taken into account while determining the best placement and capacity for DGs.

b) To assess the findings obtained in step (a), the power loss and bus voltage profile are obtained by integrating the DG units for case1, case2, and case3 in the EDN.

c) For EDN, reliability indices calculated using two different DGs reliability data, including failer rate $(\lambda i)$ and repair time ( $r i)$.

Table 2 shows how the approaches performed to reduce actual power losses and enhance voltage profiles in three cases. For comparison and analysis. Adding a single DG unit with the best size and location is (1780KW) at bus 11 , the active power losses are decreased to $(95 \mathrm{KW})$ with minimum voltage is increased to $(0.943 \mathrm{p} . \mathrm{u})$, and the voltage square error is reduced to (0.024 p.u).

Adding two DG units with the best sizes and locations is $(700 \mathrm{KW})$ at bus 13 and $(115 \mathrm{~kW})$ at bus 31. This configuration lowers the active power losses to $(89 \mathrm{~kW})$, a $(57.8 \%)$, and the minimum voltage is increased to $(0.96 \mathrm{p} . \mathrm{u})$, and the voltage square error is reduced to $(0.0219$ p.u). At adding three DG units with the best sizes and locations are $(700 \mathrm{KW})$ at bus $15,(370 \mathrm{~kW})$ at bus 30 , and $(1000 \mathrm{KW})$ at bus 33 , the active power losses are decreased to $(82 \mathrm{KW})$, a $(60.13 \%)$ reduction, the minimum voltage is increased to $(0.97 \mathrm{p} . \mathrm{u})$, and the voltage square error is reduced to (0.0156p.u). Fig. 5 shows that the active power losses reduction as percents for three cases test system. Similarly, Fig. 6 depicts the effect of the proposed approach on the voltage square error reduction. Fig. 7 shows the voltage profiles for three cases in which the proposed method has a positive effect on the voltage profiles.

The new MPSO approach allows for easy convergence toward the optimum solution, as well as quick, almost linear convergence and control of the explosion caused by particle velocity randomness.

The classical PSO is enhanced by linear decreasing of the inertia weights. This linear decrease of inertia weights is used to get the preferable solution. This modification can ensure convergence, find better and better points in the search space, and its relative weighting varies randomly with each iteration. As compared the proposed method result with the proposed in ref. [26] shows that the new MPSO provides the optimum balance between voltage profiles, power loss reduction, and reliability enhancement. 
Table 2. Results of the best placement and sizing of DGs for test systems

\begin{tabular}{|c|c|c|c|c|c|c|c|c|}
\hline \multicolumn{2}{|c|}{ Item } & $\begin{array}{c}\text { DGs } \\
\text { Location }\end{array}$ & $\begin{array}{c}\text { DGs Size } \\
\text { (MW) }\end{array}$ & $\begin{array}{l}\text { Plosses } \\
\text { (MW) }\end{array}$ & $\begin{array}{l}\text { \%Plosses } \\
\text { Reduction }\end{array}$ & $\begin{array}{c}\text { Verror } \\
\text { (p.u) }\end{array}$ & $\begin{array}{l}\text { \% Verror } \\
\text { Reduction }\end{array}$ & $\begin{array}{c}\text { Vworst } \\
\text { (p.u) }\end{array}$ \\
\hline \multicolumn{2}{|c|}{ Without DGs } & ---- & ---- & 0.2110 & ---- & 0.1338 & ---- & 0.904 \\
\hline \multicolumn{2}{|c|}{ MPSO / Case1 } & 11 & 1.78 & 0.095 & 54.97 & 0.024 & 82 & 0.943 \\
\hline LSF & \multirow{4}{*}{ Ref.(26) } & 6 & 1.2 & 0.1387 & 34.21 & 0.0803 & 40 & 0.9221 \\
\hline GA & & 12 & 1.2 & 0.1259 & 40.31 & 0.0426 & 68.15 & 0.9347 \\
\hline PMC & & 13 & 1.2 & 0.1294 & 38.62 & 0.0384 & 71.28 & 0.9347 \\
\hline PPBIL & & 13 & 1.2 & 0.1294 & 38.62 & 0.0384 & 71.28 & 0.9347 \\
\hline \multicolumn{2}{|c|}{ MPSO / Case2 } & 13,31 & $0.7,1.15$ & 0.089 & 57.8 & 0.0219 & 83.63 & 0.960 \\
\hline LSF & \multirow{4}{*}{ Ref.(26) } & 6,28 & $0.4739,1.0964$ & 0.1180 & 44.04 & 0.0598 & 55.27 & 0.9277 \\
\hline GA & & 16,32 & $0.7984,0.7719$ & 0.0954 & 54.77 & 0.0254 & 80.99 & 0.9603 \\
\hline PMC & & 15,30 & $0.7989,0.7714$ & 0.0938 & 55.53 & 0.0275 & 79.44 & 0.9552 \\
\hline PPBIL & & 14,32 & $0.8721,0.6982$ & 0.0938 & 55.50 & 0.0258 & 80.70 & 0.9590 \\
\hline \multicolumn{2}{|c|}{ MPSO / Case3 } & $15,30,33$ & $0.7,0.37,1$ & 0.082 & 60.13 & 0.0156 & 88.34 & 0.970 \\
\hline LSF & \multirow{4}{*}{ Ref.(26) } & $6,28,8$ & $0.0001,0.6343,0.9355$ & 0.1060 & 49.73 & 0.0472 & 64.66 & 0.940 \\
\hline GA & & $14,30,32$ & $0.3203,0.5258,0.2404$ & 0.0917 & 56.49 & 0.0276 & 79.31 & 0.9572 \\
\hline PMC & & $12,18,31$ & $0.4993,0.3966,0.6744$ & 0.0916 & 56.57 & 0.0266 & 80.08 & 0.9578 \\
\hline PPBIL & & $12,15,31$ & $0.4035,0.5245,0.6422$ & 0.0915 & 56.60 & 0.0265 & 80.16 & 0.9570 \\
\hline
\end{tabular}

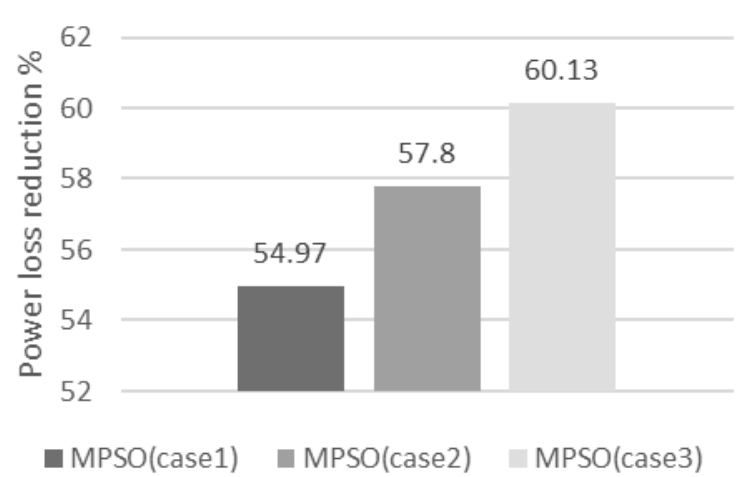

Figure. 5 Real power loss reduction

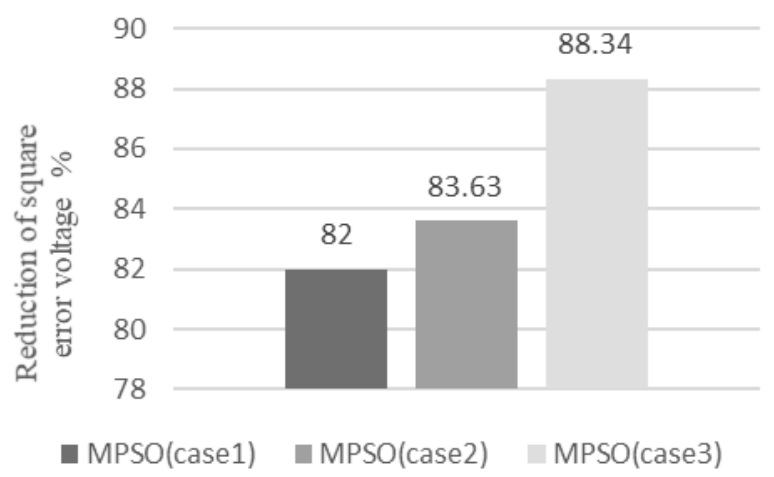

Figure. 6 Voltage profiles square error reduction

\subsection{Reliability assessment}

There are two factors in the reliability data of DGs used to determine the reliability indices. These indices are based on two reliability data: the system's elements' failer rate $(\lambda i)$ and repair time $\left(r_{i}\right)$. Considered a different reliability data for DGs only in the present work as following scenarios:

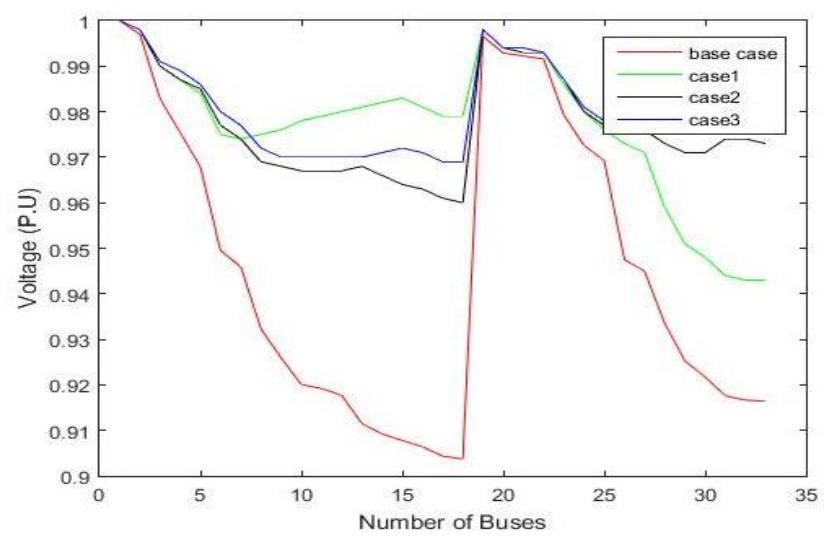

Figure. 7 Voltage profiles for 33 buses

1. Scenario \#1: $0.2 \mathrm{f} / \mathrm{yr}$ and $12 \mathrm{~h}$

2. Scenario \#2: $0.4 \mathrm{f} / \mathrm{yr}$ and $12 \mathrm{~h}$

3. Scenario \#3: $0.6 \mathrm{f} / \mathrm{yr}$ and $12 \mathrm{~h}$

4. Scenario \#4: $0.2 \mathrm{f} / \mathrm{yr}$ and $24 \mathrm{~h}$

5. Scenario \#5: $0.2 \mathrm{f} / \mathrm{yr}$ and $48 \mathrm{~h}$

6. Scenario \#6: No failure

The above six scenarios were applied to three indicators: SAIDI, SAIFI and EENS, whose value was affected by changing the repair time $\left(r_{i}\right)$ and the average failure rate $(\lambda i)$ of the DGs. For assessment of the distribution system's reliability, the following main assumptions are taken into account.

a) Circuit breaker (CB), fuse, distribution line, and potential transformer are available throughout $100 \%$ reliability.

b) Table 3 displays the average failer rate and repair time of feeders, buses, and substations [33] in Appendix A.

c) Table 4 displays the Load distribution for 33 buses [33] in Appendix A. 
Table 5. Reliability indices results of the EDN

\begin{tabular}{|c|c|c|c|c|}
\hline Item & $\begin{array}{c}\text { Base } \\
\text { case }\end{array}$ & Case 1 & Case 2 & Case 3 \\
\hline SAIDI & 23.2488 & 18.9444 & 11.6354 & 9.0630 \\
\hline SAIFI & 2.4967 & 1.8323 & 1.1517 & 0.9281 \\
\hline EENS & 82.804 & 68.594 & 43.771 & 34.561 \\
\hline AENS & 0.0265 & 0.0220 & 0.0140 & 0.0111 \\
\hline ASAI & 0.9973 & 0.9978 & 0.9987 & 0.9990 \\
\hline
\end{tabular}

Table 6. SAIDI, SAIFI, and EENS were evaluated for different scenarios

\begin{tabular}{|c|c|c|c|c|c|c|}
\hline \multicolumn{7}{|c|}{ SAIDI } \\
\hline Item & Scenario1 & Scenario2 & Scenario3 & Scenario4 & Scenario5 & Scenario6 \\
\hline Base case & 23.2488 & 23.2488 & 23.2488 & 23.2488 & 23.2488 & 23.2488 \\
\hline Case 1 & 18.9444 & 18.9456 & 18.9468 & 18.9456 & 18.9480 & 18.9432 \\
\hline Case 2 & 11.6354 & 11.6382 & 11.6410 & 11.6382 & 11.6439 & 11.6325 \\
\hline Case 3 & 9.0630 & 9.0633 & 9.0638 & 9.0640 & 9.0634 & 9.0625 \\
\hline \multicolumn{7}{|c|}{ SAIFI } \\
\hline Item & Scenario1 & Scenario2 & Scenario3 & Scenario4 & Scenario5 & Scenario6 \\
\hline Base case & 2.4967 & 2.4967 & 2.4967 & 2.4967 & 2.4967 & 2.4967 \\
\hline Case 1 & 1.8323 & 1.8326 & 1.8329 & 1.8323 & 1.8323 & 1.8321 \\
\hline Case 2 & 1.1517 & 1.1523 & 1.1528 & 1.1517 & 1.1517 & 1.1512 \\
\hline Case 3 & 0.9281 & 0.9292 & 0.9294 & 0.9281 & 0.9281 & 0.9278 \\
\hline \multicolumn{7}{|l|}{ EENS } \\
\hline Item & Scenario1 & Scenario2 & Scenario3 & Scenario4 & Scenario5 & Scenario6 \\
\hline Base case & 82.804 & 82.804 & 82.804 & 82.804 & 82.804 & 82.804 \\
\hline Case 1 & 68.594 & 68.598 & 68.602 & 68.598 & 68.606 & 68.591 \\
\hline Case 2 & 43.771 & 43.780 & 43.790 & 43.780 & 43.799 & 43.761 \\
\hline Case 3 & 34.561 & 34.566 & 34.567 & 34.566 & 34.568 & 34.551 \\
\hline
\end{tabular}

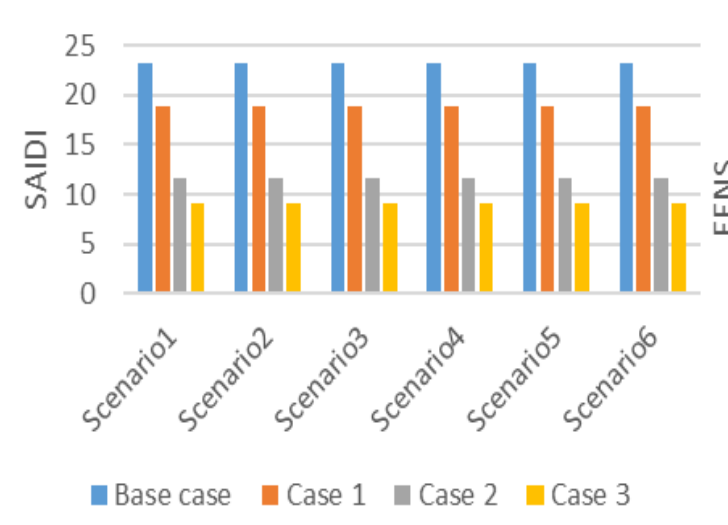

(a)
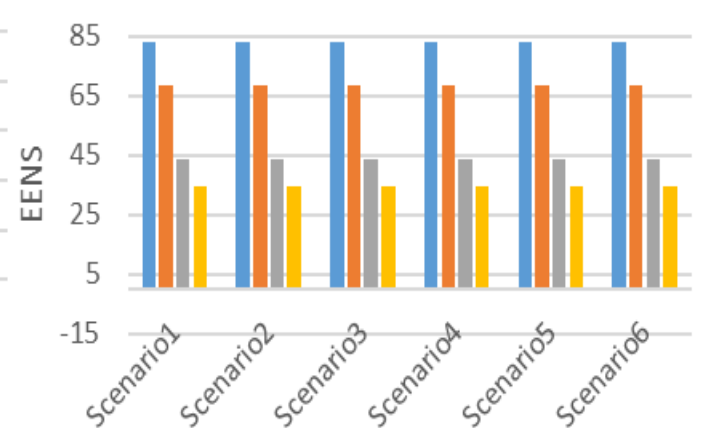

- Base case nCase 1 - Case 2 Case 3

(b)

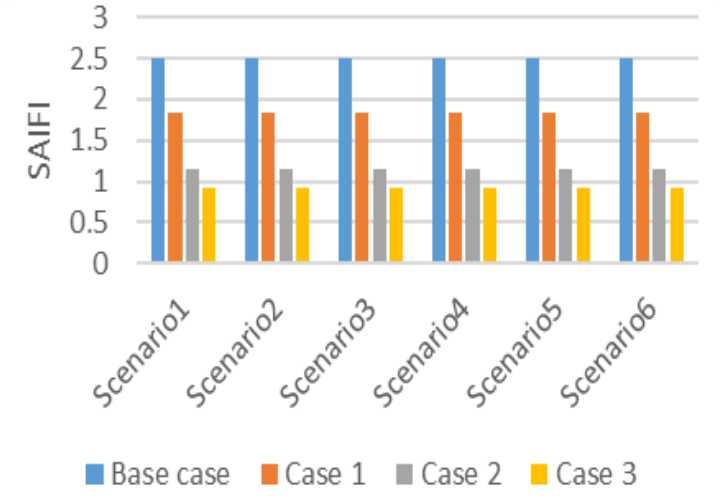

(c)

Figure. 8 Reliability indices for different DGs $\lambda i$ and $r_{i}$ : (a) SAIDI, (b) SAIFI, and (c) EENS 
The IEEE 33-bus distribution system is shown in Fig. 9, was already selected for analysis by using ETAP Models in Appendix A.

Table 5 shows results for the reliability indices of the EDN before and after adding DGs for the three cases mentioned above with $12 \mathrm{~h}\left(r_{i}\right)$ and $0.2 \mathrm{f} / \mathrm{yr}(\lambda i)$ for DGs reliability data. The addition of one DG reduced the SAIDI, SAIFI, EENS, and AENS indices to (18.9444), (1.8323), (68.594), and (0.0220), respectively and increased the ASAI index to (0.9978). Also, adding two DG resulted in reducing the SAIDI, SAIFI, EENS, and AENS indices to (11.6354), (1.1517), (43.771), and (0.0140), respectively and increased the ASAI index to (0.9987). Adding three DGs resulted in reducing the SAIDI, SAIFI, EENS, and AENS indices to (9.0630), (0.9281), (34.561), and (0.0111), respectively and increased the $A S A I$ index to (0.9990). Table 6 shows the SAIDI, SAIFI, and EENS results for three cases based on the six scenarios. Fig. 8 (a)-(c), shows these indicators for all cases based on the six scenarios. As the number of DGs units grows, these indices are reduced except the ASAI index. The increasing number of DGs is incorporated into the EDN, the length of the interruption, the number of interruptions that occurred decreases, and improves the supplied energy in the distribution system. This improvement in the supplied energy will decrease the EENS and $A E N S$ linked to the energy not supplied. It should be noted that the $\left(r_{i}\right)$ of the DGs does not impact the SAIFI. This index, as shown in Eq. (31), is independent of $\left(r_{i}\right)$. When DGs integrate into EDN, the $A S A I$ index for all loads rises. As the ASAI grows, the average system utility index ASUI decreases, as shown in Eqs. (34) and (37), which is beneficial for distribution system reliability.

\section{Conclusion}

The proposed algorithm was used for one, two, and three DGs to find optimal DGs placement and size for real power minimization, voltage profile improvement, and reliability enhancement. As compared to [26], the total real power losses are minimized by $(0.0309 \mathrm{MW}),(0.0048 \mathrm{MW})$, and $(0.0095 \mathrm{MW})$ for one, two, and three GDs, respectively. Compared to the base case, the worst value of bus voltage is increased by $(6.6 \%)$ for three DGs.

The reliability enhancement for the DGs integrated delivery system is conducted after satisfactory results have been achieved. The integration of three DGs and scenario \#6 yields the best system reliability results. After all, scenario \#6 is ideal. Case \#3 with scenario \#1 is thought to produce better results in improving the distribution system's reliability. As compared to the base case, the SAIDI index is minimized by (4.3044), (11.6134), and (14.1858), SAIFI index is minimized by $(0.664)$, (1.345), and (1.5686) and, EENS index is minimized by (14.21), (39.033), and (4.243), AENS index is minimized by (0.0045), (0.0125), and (0.0154) and ASAI index is maximized by (0.0005), (0.0014), and (0.0017) for one, two, and three DGs, respectively.

This paper will assist researchers in determining an appropriate method for reducing power loss, improving voltage profile and distribution system reliability. There are numerous research avenues to pursue in the future, like Incorporates renewable energy sources and their impact on EDN reliability. EDN reliability can also be improved by adjusting the number of branches, a method known as system reconfiguration, and its impact on distribution system reliability.

\section{Conflicts of Interest}

The authors confirm that there is no conflict of interest.

\section{Author Contributions}

"Conceptualization, Mohammed Kdair Abd and Ali Mohammed Jaleel; methodology, Mohammed Kdair Abd; software, Ali Mohammed Jaleel; validation, Mohammed Kdair Abd; formal analysis, Mohammed Kdair Abd and Ali Mohammed Jaleel; investigation, Ali Mohammed Jaleel; resources, Ali Mohammed Jaleel; data curation, Mohammed Kdair Abd; writing - original draft preparation, Ali Mohammed Jaleel; writing-review and editing, Mohammed Kdair Abd; visualization, Mohammed Kdair Abd; supervision, Mohammed Kdair Abd; project administration, Ali Mohammed Jaleel".

\section{References}

[1] A. Mohammed and S. Shahl, "Impact of Distributed Generation on a Distribution Network Voltage Sags in Baghdad City", Engineering and Technology Journal, Vol. 39, No. 4A, pp. 528-542, 2021.

[2] M. Nasser, I. Ali, and M. Alkhafaji, "Optimal Placement and Size of Distributed Generators Based on Autoadd and PSO to Improve Voltage Profile and Minimize Power Losses", Engineering and Technology Journal, Vol. 39, No. 3A, pp. 453-464, 2021.

[3] Z. Dawood and R. A. Rubayi, "Analysis of Distribution System Reconfiguration under Different Load Demand in AL-KUT City by 
using PSO Algorithm", Engineering and Technology Journal, Vol. 39, No. 5A, pp. 738753, 2021.

[4] A. A. E. Motaleb and S. Bekdach, "Optimal sizing of distributed generation considering uncertainties in a hybrid power system", International Journal of Electrical Power \& Energy Systems, Vol. 82, pp. 179-188, 2016.

[5] R. A. Rubayi, M. Abd, and F. Flaih, "A New Enhancement on PSO Algorithm for Combined Economic-Emission Load Dispatch Issues", International Journal of Intelligent Engineering and Systems, Vol. 13, No. 1, pp. 77-85, 2020.

[6] M. Abd, S. Cheng, and H. Sun, "Optimal DG placement and sizing for power loss reduction in a radial distribution system using MPGSA and sensitivity index method", In: Proc. of 2016 IEEE 11th Conf. on Industrial Electronics and Applications (ICIEA), pp. 1579-1585, 2016.

[7] A. Tabares, G. M. Delgado, J. Franco, J. Arroyo, and J. Contreras, "An enhanced algebraic approach for the analytical reliability assessment of distribution systems", IEEE Transactions on Power Systems, Vol. 34, No. 4, pp. 2870-2879, 2019.

[8] L. Bai, T. Jiang, F. Li, H. Chen, and X. Li, "Distributed energy storage planning in soft open point based active distribution networks incorporating network reconfiguration and DG reactive power capability", Applied Energy, Vol. 210, pp.1082-1091, 2018.

[9] U. Umar, G. Setyawan, F. Faanzir, F. Firdaus, A. Soeprijanto, and O. Penangsang, "New Enhanced Symbiotic Organisms Search for Optimal Location and Sizing of Distributed Generation in Radial Distribution System", International Journal of Intelligent Engineering and Systems, Vol. 13, No. 5, pp. 170-180, 2020.

[10] M. Akbari, J. Aghaei, M. Barani, T. Niknam, S. Ghavidel, H. Farahmand, M. Korpas, and L. Li, "Convex models for optimal utility-based distributed generation allocation in radial distribution systems", IEEE Systems Journal, Vol. 12, No. 4, pp. 3497-3508, 2018.

[11] S. Essallah, A. Khedher, and A. Bouallegue, "Integration of distributed generation in electrical grid: Optimal placement and sizing under different load conditions", Computers \& Electrical Engineering, Vol. 79, pp. 106461, 2019.

[12] R. G. Blanco, P. Díez, D. Borzacchiello, and F. Chinesta, "A reduced order modeling approach for optimal allocation of distributed generation in power distribution systems", In: Proc. of 2016 IEEE International Energy Conf.
(ENERGYCON), pp. 1-6, 2016.

[13] M. Suresh and E. Belwin, "Optimal DG placement for benefit maximization in distribution networks by using Dragonfly algorithm", Renewables: Wind, Water, and Solar, Vol. 5, No. 1, pp. 1-8, 2018.

[14] M. Duong, T. Pham, T. Nguyen, A. Doan, and H. Tran, "Determination of optimal location and sizing of solar photovoltaic distribution generation units in radial distribution systems", Energies, Vol. 12, No. 1, p. 174, 2019.

[15] A. E. Blasco, M. Prodanovic, R. Segovia, and E. Castronuovo, "Reliability Evaluation of GridConnected Microgrids with High Penetration of Renewable Distributed Energy Resources", In: Proc. of the CIRED Workshop, 2018.

[16] Y. Kifle, B. Khan, and P. Singh, "Assessment and enhancement of distribution system reliability by renewable energy sources and energy storage", Journal of Green Engineering, Vol. 8, No. 3, pp. 219-262, 2018.

[17] K. Zou, A. Agalgaonkar, K. Muttaqi, and S. Perera, "Distribution System Restoration With Renewable Resources for Reliability Improvement Under System Uncertainties", IEEE Transactions on Industrial Electronics, Vol. 67, No. 10, pp. 8438-8449, 2019.

[18] P. Meera and S. Hemamalini, "Reliability based optimal DG planning for a meshed distribution network", Journal of Renewable and Sustainable Energy, Vol. 11, No. 6, p. 066301, 2019.

[19] M. Ndawula, S. Djokic, and I. H. Gil, "Reliability enhancement in power networks under uncertainty from distributed energy resources", Energies, Vol. 12, No. 3, pp. 531, 2019.

[20] A. Verma and K. S. Swarup, "Optimal Placement of DG in Active Distribution System Incorporating Performance Based Rates", In: Proc. of 2018 Clemson University Power Systems Conf. (PSC), pp. 1-6, 2018.

[21] M. Dixit, P. Kundu, and H. Jariwala, "Integration of distributed generation for assessment of distribution system reliability considering power loss, voltage stability and voltage deviation", Energy Systems, Vol. 10, No. 2, pp. 489-515, 2019.

[22] C. Patel, K. Mistry, and R. Roy, "Loss Allocation in Radial Distribution System with Multiple DG Placement using TLBO", In: Proc. of 2015 IEEE International Conference on Electrical, Computer and Communication Technologies (ICECCT), pp. 5-7, 2015.

[23] S. Ahmad, S. Sardar, A. Asar, and B. Noor, 
"Impact of distributed generation on the reliability of local distribution system", International Journal of Advanced Computer Science and Applications, Vol. 8, No. 6, pp. 375382, 2017.

[24] Y. Kumar and N. Kumar, "Optimal allocation of distribution generation units in radial distribution systems using nature inspired optimization techniques", In: Proc. of 2018 International Conf. on Power, Energy, Control and Transmission Systems (ICPECTS). IEEE, pp. 1-6, 2018.

[25] A. Ali, A. Youssef, T. George, and S. Kamel, "Optimal DG allocation in distribution systems using Ant lion optimizer", In: Proc. of 2018 International Conf. on Innovative Trends in Computer Engineering (ITCE). IEEE, pp. 324331, 2018.

[26] L. G. Noreña, D. G. Montoya, and C. R. Paja, "Optimal sizing and location of distributed generators based on PBIL and PSO techniques", Energies, Vol. 11, No. 4, pp. 101, 2018.

[27] A. SalimonSA, F. Fajuke, and K. Suuti, "Load flow analysis of nigerian radial distribution network using backward/forward sweep technique", Journal of VLSI Design and its
Advancement, Vol. 2, No. 3, pp. 1-11, 2019

[28] I. Quadri, S. Bhowmick, and D. Joshi, "A comprehensive technique for optimal allocation of distributed energy resources in radial distribution systems", Applied Energy, Vol. 211, pp. 1245-1260, 2018.

[29] J. Kennedy and R. Eberhart, "particle swarm optimization", In: Proc. of ICNN'95international conf. on neural networks., Vol. 4, pp. 1942-1948, 1995.

[30] M. Clerc and J. Kennedy, "The particle swarmexplosion, stability, and convergence in a multidimensional complex space", IEEE Transactions on Evolutionary Computation, Vol. 6, No. 1, pp. 58-73, 2002.

[31] D. Transmission, "Ieee guide for electric power distribution reliability indices", IEEE Std, pp. 1366-2003, 2003.

[32] I. Association, "IEEE 1366-2012-IEEE Guide for Electric Power Distribution Reliability Indices", New York: IEEE, 2012.

[33] S. Kumar, K. Sachin, A. Vardhan, R. Elavarasan, R. Saket, and N. Das, "Reliability assessment of wind-solar PV integrated distribution system using electrical loss minimization technique", Energies, Vol. 13, No. 21, pp. 5631, 2020.

\section{Appendix}

Table 3. Reliability data for 33-bus

\begin{tabular}{|l|c|c|}
\hline \multicolumn{1}{|c|}{ Bus, Feeder, etc. } & $\boldsymbol{\lambda} \boldsymbol{i}(\mathbf{f} / \mathbf{y r})$ & $\boldsymbol{r}_{\boldsymbol{i}(\mathbf{h})}$ \\
\hline Load@4 & 0.321 & 11.04 \\
\hline Load@(5, 7-12, 29, 30, 14, 16, 18-22, 25-28) & 0.301 & 11.44 \\
\hline Load@(13, 15) & 0.314 & 11.17 \\
\hline Load@(17, 23, 24) & 0.208 & 1.75 \\
\hline Load@(31-33) & 0.327 & 10.96 \\
\hline substation & 0.1 & 5 \\
\hline feeder (2, 3, 6) & 0.2 & 3 \\
\hline
\end{tabular}

Table 4. A load of distribution for 33-bus

\begin{tabular}{|c|c|c|}
\hline Bus No. or Load Point & No. of Loads & Type of Load \\
\hline $2-5$ & 148 & Industrial \\
\hline $6-9$ & 10 & Commercial \\
\hline 11,12 & 132 & Commercial \\
\hline $13-15$ & 110 & Residential \\
\hline 16 & 2 & Residential \\
\hline $17-20$ & 118 & Residential \\
\hline $21-26$ & 126 & Residential \\
\hline $27-31$ & 108 & Residential \\
\hline 32,33 & 58 & Residential \\
\hline
\end{tabular}




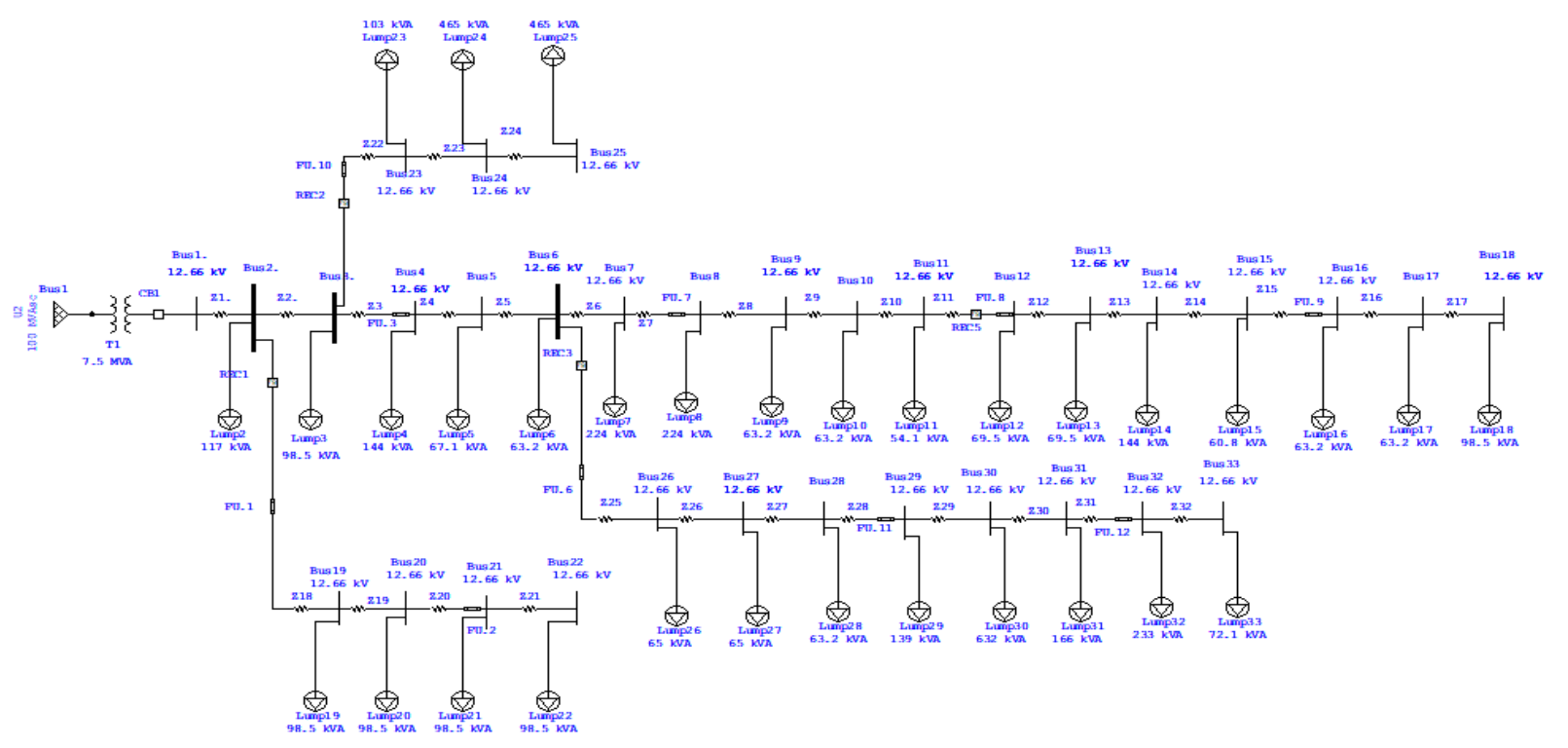

Figure. 9 Test system modelled in ETAP 